\title{
Neural Network Approach to Modelling the Behaviour of Ionic Polymer-Metal Composites in Dry Environments
}

\author{
Andrés Díaz Lantada, Pilar Lafont Morgado, José Luis Muñoz Sanz, Juan Manuel Muñoz Guijosa, \\ Javier Echávarri Otero
}

Mechanical Engineering Department_-E.T.S.I.I. Industriales, Universidad Politécnica de Madrid, Madrid, Spain.

Email: adiaz@etsii.upm.es

Received November $28^{\text {th }}, 2011$; revised December $30^{\text {th }}, 2011$; accepted January $13^{\text {th }}, 2012$

\begin{abstract}
Ionic polymer-metal composites (IPMCs) are especially interesting electroactive polymers because they show large a deformation in the presence of a very low driving voltage (around $1-2 \mathrm{~V}$ ) and several applications have recently been proposed. Normally a humid environment is required for the best operation, although some IPMCs can operate in a dry environment, after proper encapsulation or if a solid electrolyte is used in the manufacturing process. However, such solutions usually lead to increasing mechanical stiffness and to a reduction of actuation capabilities. In this study we focus on the behaviour of non-encapsulated IPMCs as actuators in dry environments, in order to obtain relevant information for design tasks linked to the development of active devices based on this kind of smart material. The non-linear response obtained in the characterisation tests is especially well-suited to modelling these actuators with the help of artificial neural networks (ANNs). Once trained with the help of characterisation data, such neural networks prove to be a precise simulation tool for describing IPMC response in dry environments.
\end{abstract}

Keywords: Ionic Polymer-Metal Composites (IPMCs); Artificial Neural Networks (ANNs); Smart Materials; Modelling and Simulation

\section{Introduction to Ionic Polymer-Metal Composites}

Electroactive polymers (EAPs) are smart materials that usually exhibit large displacements in response to external electrical stimulation. EAPs behave similarly to biological muscles, due to their electro-mechanical coupling, and have therefore acquired the nickname of "artificial muscles". Among various classes of EAPs, ionic polymer-metal composites (IPMCs) are especially interesting because they show a large deformation in the presence of a very low voltage $(1-2 \mathrm{~V})$. Additionally, a voltage (in the range of milivolts) is generated across the surfaces when the sample is subjected to mechanical loading. Thus an IPMC has both built-in actuation and sensing capabilities.

An IPMC typically consists of a thin perfluorinated polyelectrolyte or ion-exchange membrane (e.g. Nafion) sandwiched by electroplated platinum or gold on both sides. When external voltage is applied across the IPMC, mobile positive ions are drawn to the cathode side by an electric field. As a result the cathode side expands with respect to the anode, causing an overall bending deformation of the IPMC until it reaches saturation. When alternating voltage is applied, the IPMC undergoes a bending vibration at the same frequency as the applied voltage. For additional information on the fundamentals of IPMCs, among other EAPs, some excellent studies are cited in the references section [1-3]. There are a number of dynamic processes (e.g. ion migration, water diffusion, among others) taking place in IPMC actuation and the exact mechanisms are still a subject of active research. Several behaviour models, based on the results from characterisation tests, have been proposed for simplifying design activities [4-7]. Characterisation methods have also been studied, for promoting material selection tasks and for precisely validating novel manufacturing techniques, which can serve as a basis for future standards linked to electroactive polymers $[8,9]$.

Regarding industrial applications, due to their electromechanical coupling, large bending displacement, low driving voltage, resilience and potential biocompatibility, IPMCs have been studied and proposed mainly as actuators in biomimetic robotics [10,11], medical devices $[12,13]$ and micromanipulators [14-16]. Such applications benefit greatly when the actuators are manufactured following carefully controlled stages and several studies have concentrated on optimising the composition and processing of these materials [17]. Recent studies have 
described the development of three-dimensional IPMCbased actuators for special applications [18].

Normally a humid environment is required for their best operation, although some IPMCs can operate in a dry environment, if a solid electrolyte is used in the manufacturing process or after proper encapsulation [19]. However, such solutions usually lead to increasing mechanical stiffness and to a reduction of actuation capabilities, which may require alternative electroactive actuators to be selected for several applications.

In this study we focus on the behaviour of non-encapsulated IPMCs as actuators in dry environments, in order to obtain relevant information for design tasks linked to the development of active devices based on this kind of smart material. The non-linear response obtained in the characterisation tests is especially well-suited to modelling these actuators with the help of artificial neural networks (ANNs), as explained in the following section.

Once trained with the help of characterisation data, such neural networks can prove to be a significant simulation tool for design and control activities, due to their precise description of IPMC response in dry environments. We believe that the neural network-based approach can be of help, not only for design and material selection activities, but also for real-time control activities, as a remarkable alternative to very recent proposals based on genetic algorithms or fuzzy logic [20].

\section{Artificial Neural Networks for Modelling Complex Systems}

Artificial neural networks or ANNs are designed to emulate the behaviour of biological or natural neural networks. Each artificial neuron or node in the network has connections with other neurons from which it receives or passes on information. Each connection is assigned a specific weight and each neuron exhibits an actuation threshold and a characteristic input/output function. A basic characteristic of neural networks is their ability to "learn" when subjected to a learning process. That is, by taking existing data (inputs and results) an appropriate set of weights and thresholds is reached for the network to be able to reproduce the desired behaviour.

Once trained, ANNs work as an input/output system with their ability to manage multiple outputs, which makes them particularly suited to simulating complex systems. They have marked applications in different areas such as philosophy, psychology, economics, engineering and science in general $[21,22]$. Certain materials that are especially suited to modelling and simulation when artificial neural networks are used are the so-called multifunctional, active or "smart" materials that are capable of responding in a controllable manner to different external physical or chemical stimuli by modifying some of their properties (this is the case with IPMCs, as a result of their coupling electromechanical properties). Because of their sensitivity or actuation capability, these materials can be used to design and develop sensors, actuators and multifunctional systems, and having a tool that enables their behaviour to be properly simulated is of the utmost relevance [23].

The properties of these active materials assist integrating multiple functions into a system, which, combined with the major advances in micro and nanofabrication Technologies, has promoted the expansion in the last two decades of the so-called micro-electro-mechanical systems or "MEMS". These are systems based on the integration of functions and on the reduction of size in order to achieve optimised responses in time and cost for both materials and processes. Modelling these microsystems usually requires a thorough knowledge of various domains (mechanical, electrical, thermal, fluidics, magnetic...) and since their response usually exhibits marked non-linear effects, the analytical models can become too complicated to be of any practical use.

To resolve these difficulties some researchers have started to use neural networks to obtain models that enable the in-service performance of these Microsystems to be approached. An example worth pointing out is the application of ANNs to modelling (for their subsequent real-time control) a micro heat-exchanger in which the thermal, fluidic, electrical and mechanical couplings were an obstacle to obtaining an accurate analytical model [24-26].

Other researchers have also shown the usefulness of using neural networks for analysing the information furnished by sensors that monitor the behaviour of complex systems. Anomaly detection has been applied for a realtime prediction of the behaviour of the system being monitored and thus locate any problems by comparing the measurements recorded by the sensors [27,28].

Their use has also proved to be of use for processing the information from sensors (or sensor networks) that are sensitive to a range of stimuli and recognise the type of stimulus that has generated a particular response, such as previously described for mobile robots with audiotactile microsensors [29] and for therapeutic robots with multiple pressure sensors, electrical field and temperature [30].

We are of the opinion that the application of artificial neural networks to the modelling and simulation of ionic polymer-metal composites, or IPMCs, as electroactive actuators and the examination of the effects of their remaining in dry environments is a novel approach that may be an acceptable alternative to the use of complex analytical models for the design and control tasks of these smart material-based devices. The materials and methods used are described in the following sections before referring to the results from IPMC characterisa- 
tion and the subsequent training of the artificial neural networks for the response of these electroactive polymers.

\section{Materials and Methods}

\subsection{Materials}

The IPMC used to evaluate the response of this kind of material in a dry environment was supplied by Environmental Robots Inc. (www.environmental-robots.com), whose electroactive polymers stand out for the repeatability of the results, due to their using high-quality manufacturing processes, some of which are protected under patent.

According to the manufacturer, these polymeric artificial muscles are primarily thought for actuation in air but they also work immersed in water, in ionic liquids or in contact with biological tissue. Typically the bending/ flexing artificial muscles, in cantilever mode, have a tip motion of more than $10 \mathrm{~mm} / \mathrm{V}$ while drawing a maximum current of about $150 \mathrm{~mA}$. These bending/flexing IPMCs can also respond to various dynamic voltage signals such as sinusoidal, square wave, and triangular wave, among others. If they are bent, flexed or squeezed under a normal pressure, they generate a signal up to values of 2 - $4 \mathrm{mV}$.

For the characterisation tests two simples (films) of IPMC were used, coated with Pt $(5-10 \mu \mathrm{m}$ electrode thickness), in sizes of $20 \mathrm{~mm} \times 5 \mathrm{~mm} \times 0.2 \mathrm{~mm}$ and all with similar properties, each of which was characterised following the procedure set out below.

\subsection{Methods}

Each characterisation test consisted in connecting an IPMC film to a signal generator (Figure 1) so that the voltage and frequency from a square signal applied between the sides of the film could be controlled as well as the recording of the deformations attained (Figure 2).

This signal generator was purchased as part of the "Deluxe Complimentary Package" from Environmental Robots Inc. During the tests the period of the signal applied to the IPMC films was set at $14 \mathrm{~s}$, which was sufficient for the films to reach their maximum deformation. The signal had a square wave form with a half cycle at $4.5 \mathrm{~V}$ and a half cycle at $-4.5 \mathrm{~V}$, which enabled the response of these actuators to be analysed when they bent in different directions.

In preparation for the characterisation tests, the two IPMC films were kept immersed in water for 48 hours to ensure a correct homogeneous hydration. To evaluate the influence of the time passed in a dry environment on the response of the IPMC, different measurements were taken for each film that corresponded to times of 1,10 ,

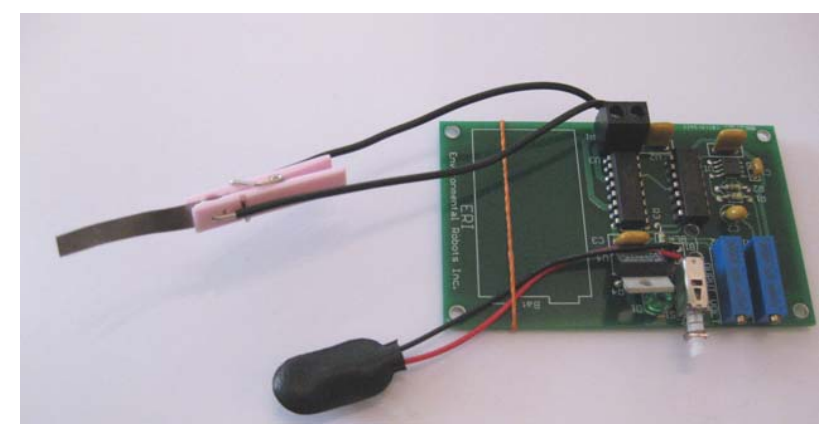

Figure 1. Kit for ionic electroactive polymer study. Supplied by Environmental Robots Inc.

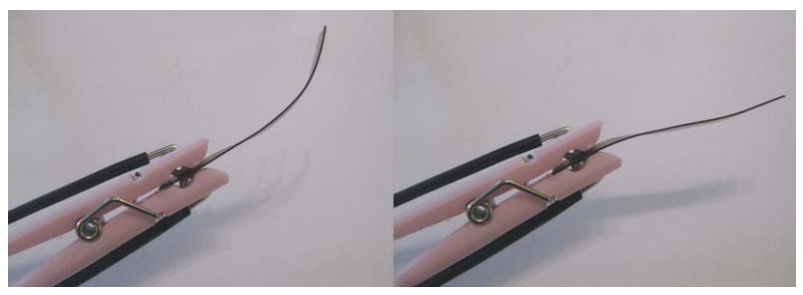

Figure 2. Activation of IPMC film. Supplied by Environmental Robots Inc.

30, 60 and 90 minutes of its being removed from the water.

The way the films are positioned is also important. It is best to place them parallel and upright so as to avoid any imbalance in the different directions of actuation as a result of their own weight. The characterisation tests were performed at a temperature of $26^{\circ} \mathrm{C}$ under a controlled relative humidity of $10 \% \pm 2 \%$. The tests were recorded on video using a digital camera so that the results could then be evaluated.

\subsection{Support Software}

Although different commercial software is available that is specifically intended for the construction, training and simulation of artificial neural networks, (Stuttgart Neural Network Simulator, Emergent, JavaNNS, Neural Lab, Genesis and others), we chose to use Matlab ${ }^{\circledR}$ R2009b as support software.

Matlab $^{\circledR}$ (The Mathworks Inc.) is a high level programming language that is equipped with an interactive environment that helps it carry out complex calculation tasks more simply and directly than using traditional programming languages. It is widely-used in universities, in part due to the ease with which information can be exchanged with other data analysis programs and spread sheets like Excel and even other programming languages like $\mathrm{C}$ or Java.

It is also closely linked to the powerful Simulink ${ }^{\circledR}$ tool, a multi-domain simulation platform based on dynamic and embedded systems models. Simulink ${ }^{\circledR}$ comes with an interactive graphic environment and a set of customis- 
able block libraries that allow designing, simulating, implementing and testing a wide variety of systems with time variation. These include mechanical systems and communication, control, signal processing and video and image systems. It is especially suitable for analysing the effects of modifying the different factors that influence the behaviour of complex systems due to the fact that the global system can be divided into simpler subsystems (in the form of blocks with inputs and outputs).

For this study we have used Matlab ${ }^{\circledR}$ R2009b because it has the major advantage of including the Neural Network Toolbox ${ }^{\mathrm{TM}}$ 6.0.3. as a work tool specifically intended for use with neural networks in tasks such as the approximation of functions, pattern recognition or the identification and control of markedly non-linear systems [31]. The aforementioned library not only enables neural networks to be constructed, trained and simulated with the support of the numerous functions already explained, but also enables .m files, that can be incorporated into more complex programs, to be directly obtained as well as .mdl models that can be integrated as independent blocks into more complex simulators programmed with the aid of Simulink ${ }^{\circledR}$. Its teaching applications are also outstanding in a whole range of scientific-technological disciplines [32].

So, having set up the neural network to simulate the behaviour of the target system, in this case the IPMCbased actuators, it is easy to use in already existing programs and simulators (to analyse the most complex global system), possibly by replacing the code for an analytical expression modelled by this subsystem by the code associated with the ensuing neural network or it can also be used as an alternative to other approaches based on the use of genetic algorithms.

The results of the tests to characterise IPMC response in a dry environment and the subsequent simulation of this behaviour using artificial neural networks are detailed in the next sections before presenting the main conclusions and the proposals for follow-up.

\section{Characterisation Results for IPMC Response in a Dry Environment}

This section sets out the results of the IPMC film characterisation tests carried out in line with the methods described previously. To show the results of the tests, we defined the bending angle by taking two straight lines: the line formed on joining the end point of the IPMC film with its clamping point and the mean longitudinal line of the actuator film when at rest (with zero bending angle). The angle formed by these two straight lines forms the bending angle taken into account and the evolution of which we have included in the figures.

Figure 3 reflects how the maximum bending angle recorded by the IPMC in its actuation cycle evolves during the time it is out of water and takes account of the bending towards positive angles (upper curve), as well as the bending towards negative angles (lower curve). The mean values for the two tests performed have been taken (each one with an actuating film) and error lines have also been included that take account of the standard deviation of the measurements taken for the times evaluated outside the water.

The maximum bending in both directions presents a

IPMC Response Evolution in Dry Environment

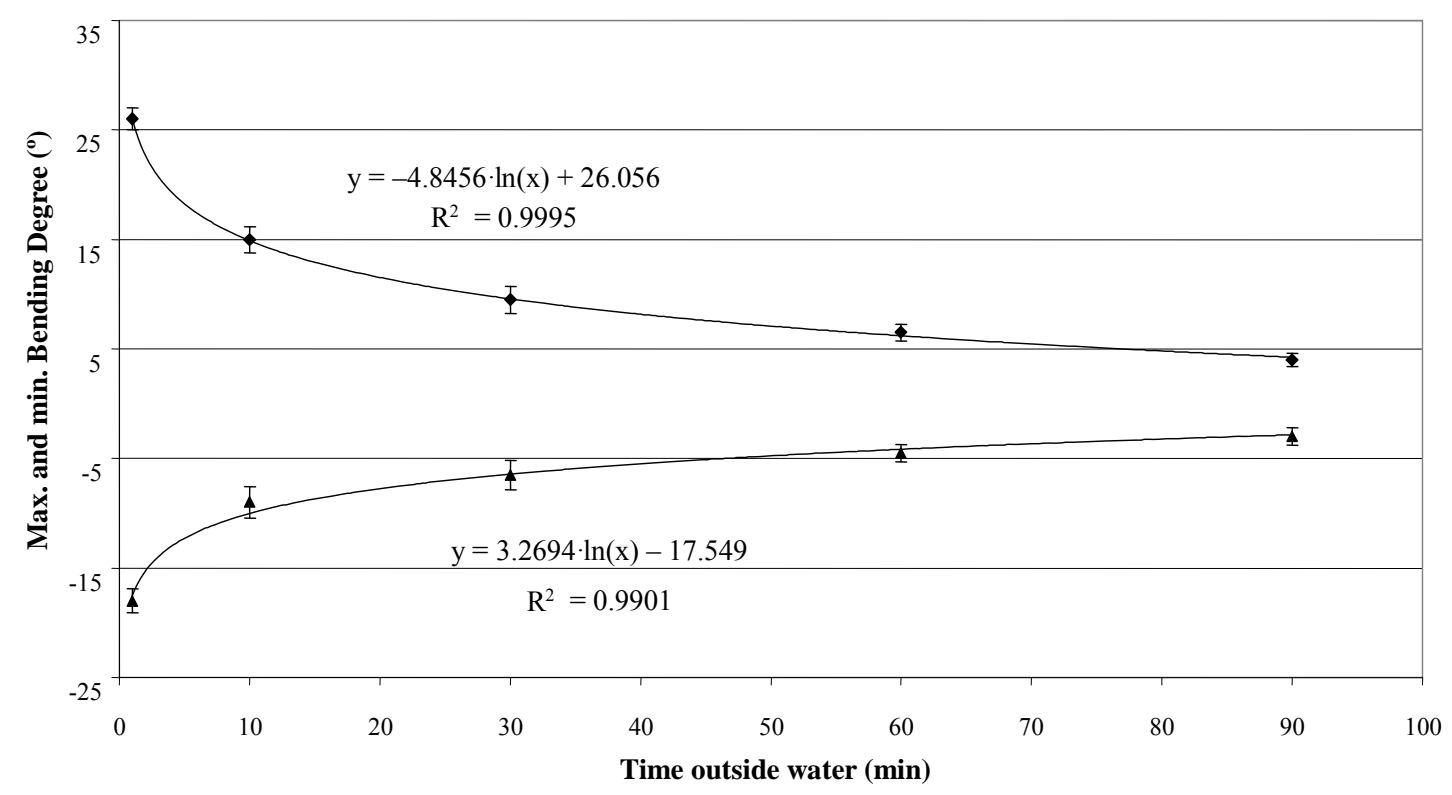

Figure 3. IPMC response outside water: evolution of maximum and minimum values for bending degree. 
markedly exponential decline with the passage of the time the actuator is out of water. Also included in Figure 3 are the equations that approximate this evolution with regression coefficient values that are close to one.

Thus, in the first 10 minutes in the open air, the actuator shows a reduction of around $50 \%$ of its maximum bending capability. After 90 minutes out of the water the maximum bending angle values recorded are less than $5^{\circ}$. This is indeed a small value for this kind of material but may be sufficient for a range of applications where it is wished to use IPMCs as micro-manipulators.

Certain asymmetries can also be observed in the behaviour of the actuator, since the angle bent towards positive values is revealed to be around $30 \%$ greater than the angle bent towards negative values, a difference that was maintained throughout the tests. We believe that these asymmetries may be due to the structure and chemical behaviour of the actuator's internal polymeric film which may favour an exchange of ions in one direction and hinder it in the other.

Figure 4 represents two full actuation cycles of an IPMC film when a square signal is applied between its sides. The signal frequency applied to the IPMC films was set at $14 \mathrm{~s}$, which is sufficient for the films to reach their maximum deformation, as can be seen from the bending angles shown. In fact, the last two pieces of bending angle data for each of the half periods shown reveal almost identical values. The signal is in the form of a square wave, with half a cycle at $4.5 \mathrm{~V}$ and half a cycle at $-4.5 \mathrm{~V}$, and has been shown as superimposed on the lines that show the evolution of the bending angles.
It can be observed that as the time passes in the dry environment, the actuator response shows a fall in amplitude (as Figure $\mathbf{3}$ has already shown) and gradually becomes slower and smoother. The cited asymmetries are clear throughout the actuation cycle with a slower response being observed towards the negative values of the bending angle than towards the positive values.

It is once again important to emphasise the need to perform thorough characterisation tests when working with any kind of smart materials for the development of new devices, as since we are usually dealing with newly available materials, their sensing and actuating properties are not usually fully known. The characterisation sheets that are generally supplied by the manufacturers often do not contain sufficiently detailed information to be able to use these materials without any additional characterisation processes. The information received often comes from theoretical simulations that must be tested in order to evaluate whether a material is viable for a specific application or device.

Therefore, in the event of using this kind of material as actuators out of water, control systems must be borne in mind right from the design stage that will let this information be included and analyse in which operating curve the IPMC is to be found, according to the time it has remained in a dry environment. To enhance the results, it may be advisable to incorporate irrigation devices (drops, sprays...) to maintain these actuators in optimal operating conditions. For this purpose, it can also be useful to use artificial neural network-based simulators to supplement theoretical models or fuzzy logic-based models, as

IPMC Response Evolution in Dry Environment

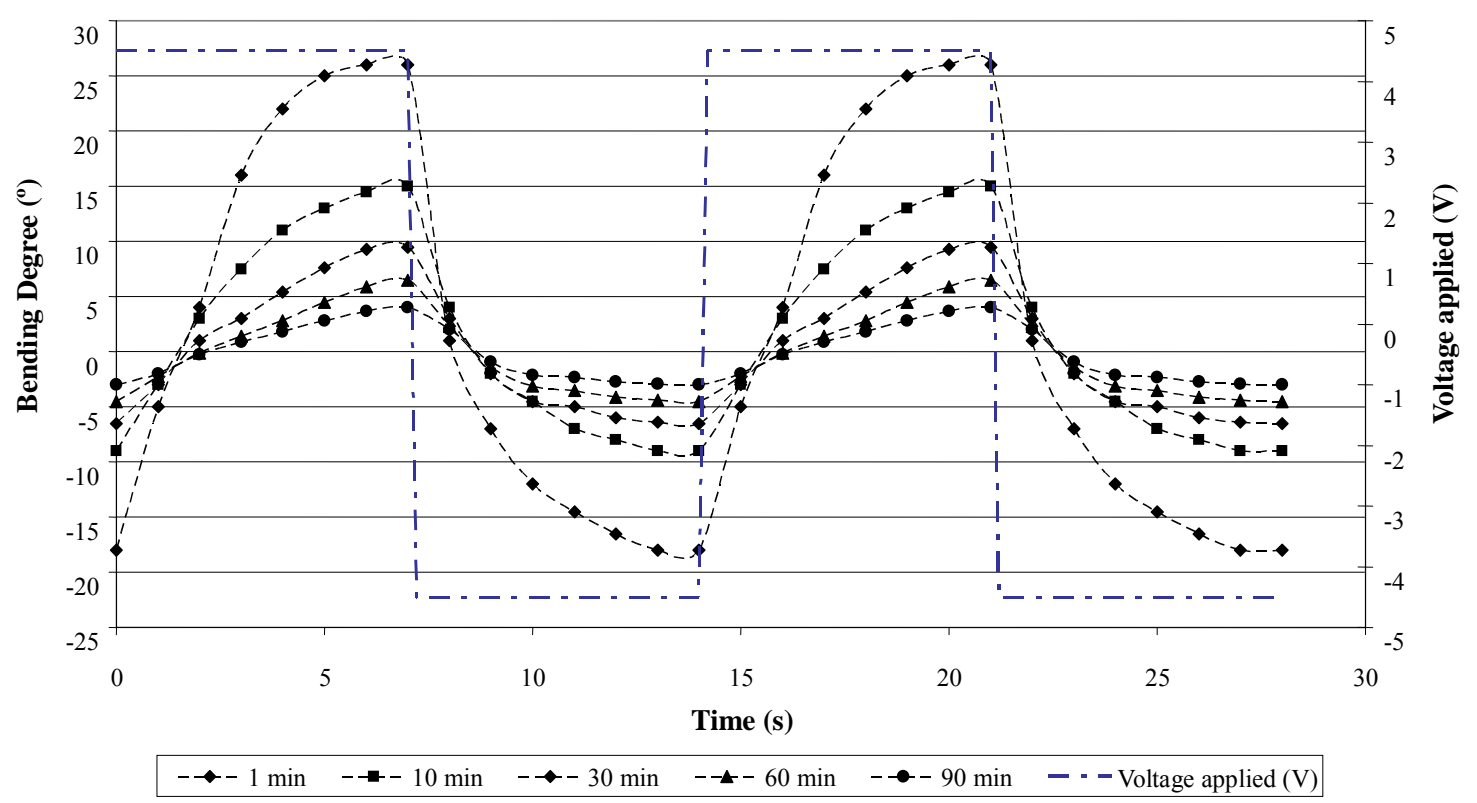

Figure 4. IPMC response evolution outside water. 
will be examined in the following section.

\section{Modelling IPMC Behaviour Using Artificial Neural Networks}

In order to prepare, train and fit the tests and validate and simulate the different neural models, the Matlab ${ }^{\circledR} \mathrm{R} 2009 \mathrm{~b}$ Neural Network Toolbox ${ }^{\mathrm{TM}}$ interactive tool was used to fit the "nftool" functions. It was decided to make a comparison of neural networks, all with 1 hidden layer and an increasing number of neurons, including values from 5 up to 100 neurons.

In the different models the input layer has 2 inputs, cycle time (seconds, for a total of $30 \mathrm{~s}$, corresponding to two bending cycles of IPMC) and the time out of water (in minutes, for a total of 90 minutes under water studied), while the results layer gives a single value or output that corresponds to the bending angle of the IPMC film. The sigmoid function was chosen as transfer function, both for the hidden layer and the results layer. It was the structure generated by Matlab ${ }^{\circledR} \mathrm{R} 2009 \mathrm{~b}$ that was charged with normalising the inputs in the range $[0,1]$, so as to optimise the calculation results with the "mapminmax" tool, which usually leads to faster learning and training (Gad-el-Hak, 2002).

The tests performed furnished 280 samples, of which $70 \%$ (146 data items) were used to train the neural networks - $15 \%$ (47 data items) as validation during the training process to analyse when it was appropriate to carry out this training and the remaining 15\% (47 data items) to test the training undergone. Training was carried out using the Levenberg-Marquardt backpropagation method. After training the neural networks, the response of each one was simulated by entering all the time cycle conditions (seconds) as inputs and all the time cycle conditions and the time the actuator was out of water (minutes), so as to be able to compare the outputs from the networks with the bending angle values measured in the tests.

The goodness of the simple linear regression among the simulation data and the test results is remarkable. In the case of 10 neurons in the hidden layer, regression coefficient values greater than 0.97 were obtained, both for the data used in the training, for the data used in the validation, and for the data used to evaluate the artificial neural network. The regression coefficient or $\mathrm{R}$ is the slope of the line obtained using linear least squares fitting for the set of points ANN estimation vs trial results, hence the nearer to 1 , the better simulation results. It is important to mention that the results are not unique since they depend on the values selected (random) by the program for the various training, validation and network testing processes. Different training processes can slightly change the results but not in any relevant way for this study. On the other hand, Figure 5 shows how the number of neurons influences the network regarding important aspects of the simulation results, such as the regression coefficient and the mean square error. It can be seen that with more than 10 neurons the quality of the results scarcely improves, whereas above 40 neurons the errors

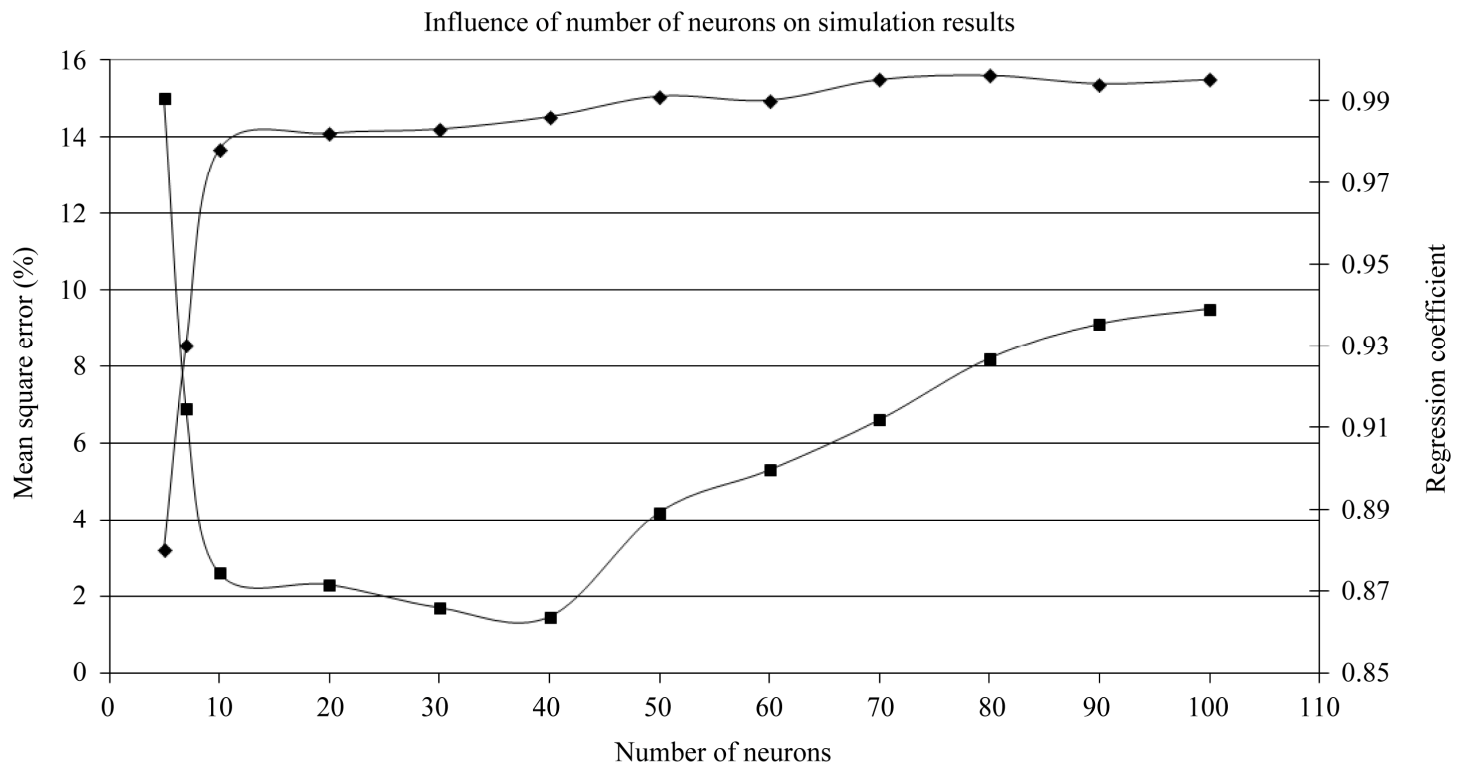

- Regression coefficient

- Mean square error

Figure 5. Influence of neuron number in the values for mean square error (\%) and regression coefficient (R), obtained when comparing results from tests and from ANN-based simulation. 
begin to rise to unacceptable figures. This, together with the increase in calculation time for larger numbers of neurons provides grounds for considering 10 to 20 neurons to be optimal numbers for this particular case study. Finally, the 10 neuron model was chosen as the most representative option and this model was used to simulate IPMC behaviour throughout two actuation cycles (28 seconds in all) for different times out of water. Simulations were performed out of water that went from 0 to 90 minutes, with 1 minute intervals between the different simulations. This enabled the information obtained in the tests to be extended and a more detailed prediction of the behaviour to be made. The simulation results are shown in Figure 6. The fit between the simulation results and the test data is satisfactory in general terms. Figure 6, resulting from the simulations performed with the artificial neural network, shows results that were to be expected according to the test data recorded in Figures 3 and 4. The possibility of using neural network as a tool for simulating IPMC behaviour is particularly promising for design tasks that require evaluating the capabilities of this material as an actuator in dry environments.

The study has enabled the use of artificial neural networks for modelling IPMC behaviour in dry environments to be validated, providing data from methodically conducted tests are used for training these networks. Indeed, the fit is fairly satisfactory, even with a small number of neurons $(10-15)$ for which regression coefficient values of $\mathrm{R} \approx 0.98-0.99$ are obtained and mean square errors of MSE $\approx 9 \%-11 \%$. The mean square error or MSE measures the average of the squares of the "errors", being the error the amount by which the value implied by the estimator (in this case artificial neural network) differs from the quantity to be estimated (in this case result from trials). If these results are compared with those of recent research based on the use of identification techniques using genetic algorithms [20], for which values of
$\mathrm{R} \approx 0.99$ and $\mathrm{MSE}<30 \%$, we can state that the use of artificial neural networks for simulating these effects constitutes a supplementary alternative that is well worth examining.

\section{Main Conclusions and Future Work}

The study has shown the important influence of the time outside an ionic liquid medium on the mechanical response of ionic polymer-metal composites when they receive an electrical stimulus. The maximum amplitude of the deformations obtained by these actuators exhibits an exponential decline which leads to a $50 \%$ reduction in their actuation capability during the first 10 minutes of their being out of water. This has considerable implications when designing electroactive actuator-based devices. Some asymmetries and non-linearities recorded in the behaviour of these smart materials and in their response in dry environments may turn out to be complex to model. This encourages the use of tools such as artificial neural networks. Once these neural networks had been properly trained by means of information from the characterisation tests, they were able to be used to simulate the behaviour of these actuators at every instant of their actuation cycle while considering a time range for being out of water that is sufficiently ample for the majority of applications put forward.

It is important to point out that the neural networkbased approach does not aim to replace the use of complex analytical models. They have been proved to be highly valuable for understanding the couplings between the thermal, electrical and mechanical properties of these materials and their subsequent response under ideal conditions. Our proposal is to use these neural models to supplement theoretical-analytical models when evaluating the behaviour of these polymer-metal composites in wide ranges of application, even in dry environments

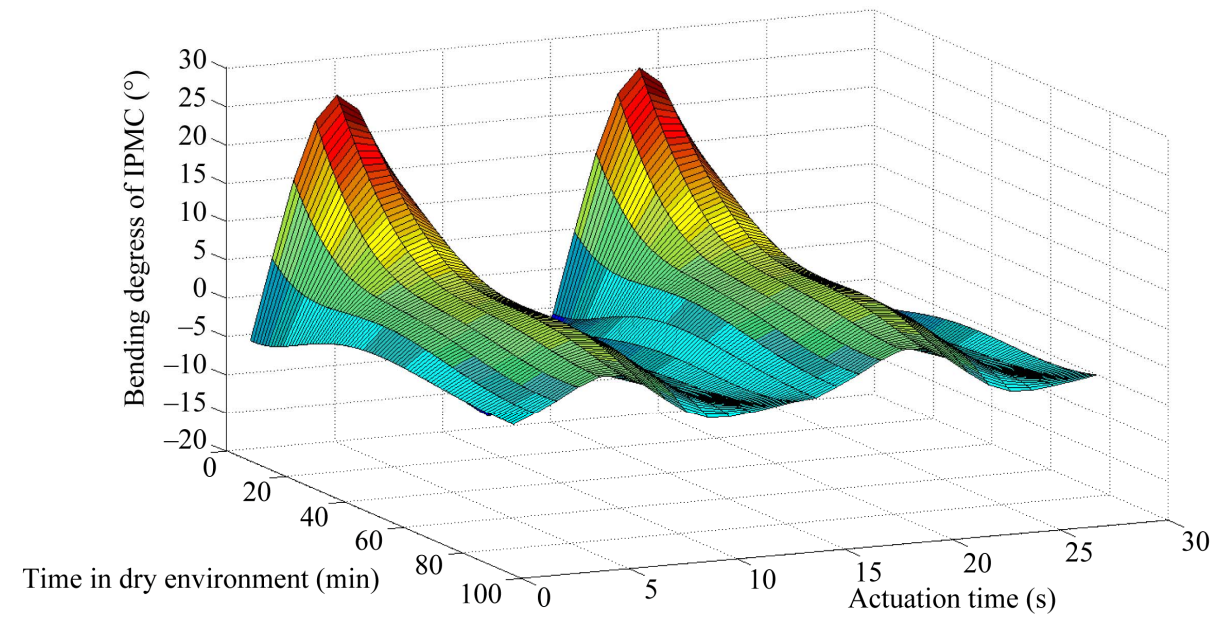

Figure 6. ANN-based simulation of IPMC response outside water (using 10 neurons in hidden layer). 
where the effects of the non-linearities are particularly marked and can lead to over-complex analytical models. Additionally, once a neural network has been created and trained, by using Matlab's Neural Network Toolbox ${ }^{\mathrm{TM}}, \mathrm{m}$ and .mdl files can be obtained directly and be integrated as sub-systems into more complex Matlab programs and Simulink models. This makes it much easier to design devices that use these materials as actuators and to compare alternatives when selecting a specific transducer.

We hope that the neural approach put forward here, together with the information from the tests performed will be of help to other researchers involved in the design tasks of new devices based on the properties of these materials as electroactive actuators. We believe it would be interesting to analyse the possible use of models with various neuron layers as a way of optimising the relation between accuracy and calculation time. It would also be interesting to carry out a more detailed study of how the results of the different fits influence the training method and the percentages of data used for training, validation and verification.

Although the work has focused on characterising, modelling and simulating IPMCs as electromechanical transducers, we believe it could also be interesting to use neural networks to simulate the global performance of future complex devices that integrate these materials into some actuator sub-system as well as for analytical tasks and their real-time control. It is our desire to be able to dedicate future efforts to these tasks and we hope to be able to collaborate with other researchers as a source of continuous improvement.

\section{REFERENCES}

[1] M. Shahinpoor and K. J. Kim, "Ionic Polymer-Metal Composites: I Fundamentals," Smart Materials and Structures, Vol. 10, No. 4, 2001, pp. 819-833. doi:10.1088/0964-1726/10/4/327

[2] Y. Bar-Cohen, S. Leary, M. Shahinpoor, J. O. Harrison and J. Smith, "Electro-Active Polymer (EAP) Actuators for Planetary Applications," Proceedings of SPIE's 6 Annual International Symposium on Smart Structures and Materials, Newport Beach, 1-5 March 1999, Paper No. 3669-05.

[3] Y. Bar-Cohen, "Electroactive Polymers as Artificial Muscles: Current Capabilities and Challenges," SPIE Press, Bellingham, 2006.

[4] S. Nemat-Nasser and J. Y. Li, "Electromechanical Response of Ionic Polymer-Metal Composites," Journal of Applied Physics, Vol. 87, No. 7, 2000, pp. 3321-3332. doi:10.1063/1.372343

[5] S. Nemat-Nasser, "Micromechanics of Actuation of Ionic Polymer-Metal Composites," Journal of Applied Physics, Vol. 92, No. 5, 2002, pp. 2899-2951. doi: $10.1063 / 1.1495888$
[6] P. Brunetto, L. Fortuna, S. Graziani and S. Strazzeri, "A Model of Ionic Polymer-Metal Composite Actuators in Underwater Operations," Smart Materials and Structures, Vol. 17, 2002, Article ID 025029.

[7] J. Choonghee, E. Hani, E. Naguib and R. H. Kwon, "Modeling and Optimization of the Electromechanical Behavior of an Ionic Polymer-Metal Composite," Smart Materials and Structures, Vol. 17, 2008, Article ID 065022.

[8] C. Bonomo, L. Fortuna, P. Giannone and S. Graziani, “A Method to Characterize the Deformation of an IPMC Sensing Membrane," Sensors and Actuators A: Physical, Vol. 123-124, 2005, pp. 146-154. doi:10.1016/j.sna.2005.03.012

[9] T. Yokota, K. Kikuchi and S. Tsuchitani, "Evaluation of Basic Operating Characteristics of Ion Conductive Polymer Actuator Using Ionic Liquid," SICE Annual Conference, Sakaedani, 20-22 August 2008, pp. 1096-1099.

[10] S. Guo, T. Fukuda and K. Asaka, "A New Type of FishLike Underwater Microrobot," IEEE/ASME Transactions on Mechatronics, Vol. 8, No. 1, 2003, pp. 136-141. doi:10.1109/TMECH.2003.809134

[11] B. Kim, J. Ryu, Y. Jeong, Y. Tak, B. Kim and J.-O. Park, "A Ciliary Based 8-Legged Walking Micro Robot Using Cast IPMC Actuators," Proceedings of IEEE International Conference on Robots and Automation, Vol. 3, 2003, pp. 2940-2945.

[12] S. Guo, T. Fukuda, K. Kosuge, F. Arai and M. Negoro, "Microcatheter System with Active Guide Wire," Proceedings of IEEE International Conference on Robotics and Automation, Vol. 1, 21-27 May 1995, pp. 79-84.

[13] H.-H. Lin, B.-K. Fang, M.-S. Ju, C. Ching and K. Lin, "Control of Ionic Polymer-Metal Composites for Active Catheter Systems via Linear Parameter-Varying Approach," Journal of Intelligent Material Systems and Structures, Vol. 20, No.3, 2009, pp. 273-282. doi: 10.1177/1045389X08093565

[14] Y. Bar-Cohen, S. Leary, M. Shahinpoor, J. O. Harrison and J. Smith, "Flexible Low-Mass Devices and Mechanisms Actuated by Electroactive Polymers," Proceedings of SPIE, Vol. 3669, 1999, pp. 51-56. doi: $10.1117 / 12.349697$

[15] S. Tadokoro, S. Yamagami and M. Ozawa, "Soft Micromanipulation Device with Multiple Degrees of Freedom Consisting of High Polymer Gel Actuators," Proceedings of IEEE International Conference on Micro Electro Mechanical Systems, 17-21 January 1999, pp. 37-42.

[16] R. Lumia and M. Shahinpoor, "Microgripper Design Using Electroactive Polymers," In: Y. Bar-Cohen, Ed., Smart Structures and Materials 1999: Electroactive Polymer Actuators and Devices, SPIE, Bellingham, 1999, pp. 322329.

[17] S. J. Lee, M. J. Han, Se. J. Kim, J. Y. Jho, H. Y. Lee and Y. H. Kim, "A New Fabrication Method for IPMC Actuators and Application to Artificial Fingers," Smart Materials and Structures, Vol. 15, No. 5, 2006, pp. 1217-1224. doi:10.1088/0964-1726/15/5/008

[18] C. Bonomo, M. Bottino, P. Brunetto, G. Di Pasquale, L. Fortuna, S. Graziani and A. Pollicino, "Tridimensional Ionic Polymer Metal Composites: Optimization of the 
Manufacturing Techniques," Smart Materials and Structures, Vol. 19, No. 5, 2010, Article ID 055002. doi:10.1088/0964-1726/19/5/055002

[19] S. J. Kim, I. T. Lee, H.-Y. Lee and Y. H. Kim, "Performance Improvement of an Ionic Polymer-Metal Composite Actuator by Parylene Thin Film Coating," Smart Materials and Structures, Vol. 15, No. 6, 2006, p. 1540.

[20] B. C. Lavu, M. P. Schoen and A. Mahajan, "Adaptive Intelligent Control of Ionic Polymer-Metal Composites," Smart Materials and Structures, Vol. 14, No. 4, 2005, pp. 466-474. doi:10.1088/0964-1726/14/4/002

[21] R. J. Schalkoff, “Artificial Neural Networks," Mc Graw Hill, New York, 1997.

[22] S. Haykin, "Neural Networks: A Comprehensive Foundation," 2nd Edition, Prentice Hall, Englewood Cliffs, 1999.

[23] A. D. Lantada, "Metodología Para el Desarrollo de Dispositivos Médicos Basados en el Empleo de Polímeros Activos Como Sensores y Actuadores," Ph.D. Thesis, Universidad Politécnica de Madrid, Madrid, 2009.

[24] G. Diaz, M. Sen, K. T. Yang and R. L. Mc Clain, "Simulation of Heat Exchanger Performance by Artificial Neural Networks," International Journal HVAC \& Research, Vol. 5, No. 3, 1999, pp. 195-208,.

[25] G. Diaz, M. Sen, K. T. Yang and R. L. Mc Clain, "Dynamic Prediction and Control of Heat Exchangers Using Artificial Neural Networks," International Journal of Heat Mass Transfer, Vol. 44, No. 9, 2001, pp. 1671-1679. doi:10.1016/S0017-9310(00)00228-3

[26] M. Gad-el-Hak, Ed., "The MEMS Handbook," CRC Press,
New York, 2002.

[27] S. Gulati and M. Zak, "Neural-Network Approach to Analysis of Sensor Data," NASA Technology Briefs, 2000.

[28] W. A. Rakowski and S. Zimowski, "Polyesterimide Composites as a Sensor Material for Sliding Bearings," Composites Part B: Engineering, Vol. 37, No. 2-3, 2006, pp. 81-88. doi:10.1016/j.compositesb.2005.09.002

[29] P. Manoonpong, F. Pasemann and J. Fischer, "Neural Processing of Auditory-Tactile Sensor Data to Perform Reactive Behaviour of Walking Machines," Proceedings of the IEEE International Conference on Mechatronics and Robotics, 3-5 June 2004.

[30] W. D. Stiehl, D. Liebermann, C. Breazeal, L. Basel, L. Lalla and M. Wolf, "Design of a Therapeutic Robot Companion for Relational, Affective Touch," Proceedings of the 2005 IEEE International Workshop on Robots and Human Interactive Communication, Cambridge, 13-15 August 2005, pp. 408-415. doi:10.1109/ROMAN.2005.1513813

[31] H. Demuth, M. Beale and M. Hagan, "Neural Networks Toolbox ${ }^{\text {TM }}$ User's Guide,” The Mathworks Inc., 1992-2009, Online Only, Revised for Version 6.0.3.

[32] E. Bautista Paz, J. Echávarri Otero, J. M. Munoz-Guijosa, A. Díaz Lantada, P. Lafont, J. M. García, J. L. Muñoz Sanz and H. L. Yustos, "Simulink Model for Teaching the Stick - Slip Friction Phenomenon in 'Machine Vibration and Noise' Course," International Journal of Engineering Education, Vol. 25, No. 2, 2009, pp. 102-111. 